TRANSACTIONS OF THE

AMERICAN MATHEMATICAL SOCIETY

Volume 349, Number 4, April 1997, Pages 1503-1514

S 0002-9947(97)01914-4

\title{
THE GROUP OF GALOIS EXTENSIONS OVER ORDERS IN $K C_{p^{2}}$
}

\author{
ROBERT UNDERWOOD
}

\begin{abstract}
In this paper we characterize all Galois extensions over $H$ where $H$ is an arbitrary $R$-Hopf order in $K C_{p^{2}}$. We conclude that the abelian group of $H$-Galois extensions is isomorphic to a certain quotient of units groups in $R \times R$. This result generalizes the classification of $H$-Galois extensions, where $H \subset K C_{p}$, due to Roberts, and also to Hurley and Greither.
\end{abstract}

\section{INTRODUCTION}

Let $K$ be a finite extension of the $p$-adic rationals $\mathbf{Q}_{p}$ endowed with the $p$-adic valuation $\nu$ with $\nu(p)=1$. Let $R$ be the integral closure of $\mathbf{Z}_{p}$ in $K$ and let $H$ be an arbitrary $R$-Hopf algebra order in $K C_{p^{2}}$. We assume that $R$ contains $\zeta$, a primitive $p^{2}$ nd root of unity; thus the linear dual $H^{*}=H_{o m}(H, R)$ is an $R$-Hopf algebra order in $K C_{p^{2}}$. In this paper we characterize all Galois extensions over $H$, and hence, all Galois algebras over $H^{*}$. We conclude that the abelian group of $H$-Galois extensions is isomorphic to a certain quotient of units groups in $R \times R$. This result generalizes the classification of $H$-Galois extensions, where $H \subset K C_{p}$, due to Roberts [R, Thm. 1], and also found in [H, Thm 4.9] and [G, Prop. II.2.1].

\section{Definitions AND PRELIMINARIES}

Let $C_{p^{2}}$ denote the cyclic group of order $p^{2}$ with generator $g$. Then the group ring $K C_{p^{2}}$ can be endowed with the structure of a $K$-Hopf algebra, with $\Delta$, $\epsilon$, and $\sigma$ denoting the co-multiplication, co-unit, and antipode maps. An $R$-Hopf algebra order in $K C_{p^{2}}$ is an $R$-Hopf algebra $H$ which is a finitely generated projective $R$ module satisfying

$$
H \otimes_{R} K \cong K C_{p^{2}}
$$

as $K$-Hopf algebras. Note that as a finitely generated module over a local ring $R$, a Hopf algebra order in $K C_{p^{2}}$ is free over $R$ of rank $p^{2}$.

The structure of $R$-Hopf algebra orders in $K C_{p^{2}}$ has been determined by C. Greither in [G, Cor. 3.6], and this author in [U2, Main Theorem]. For an arbitrary $R$-Hopf algebra order $H$ in $K C_{p^{2}}$, we have that

$$
H=A_{v}(s, r)=R\left[\frac{g^{p}-1}{x_{s}}, \frac{g-a_{v}}{x_{r}}\right], \quad\langle g\rangle=C_{p^{2}} .
$$

Received by the editors June 9, 1995

1991 Mathematics Subject Classification. Primary 14L15, 16W30, 13B02; Secondary 13B25, 11Sxx.

(C) 1997 American Mathematical Society 
Here $x_{s}, x_{r}$ denote elements in $R$ of value $s, r$ respectively. The quantity $a_{v}$ is an element in $R\left[\frac{g^{p}-1}{x_{s}}\right]$ of the form $a_{v}=\sum_{i=0}^{p-1} v^{i} e_{i}$, where $v$ is a certain unit in $R$ and the $e_{i}$ are the idempotents for the maximal integral order in $K C_{p}$ (for details see [G, Cor. 3.6], [U2, §1.2], [U3, §3.1]). Moreover, it is not difficult to show that the algebra generator $\frac{g^{p}-1}{x_{s}}$ is a root of the monic polynomial

$$
p(X)=x_{-p s}\left(\left(1+x_{s} X\right)^{p}-1\right)
$$

of degree $p$ with coefficents in $R$, and that the generator $\frac{g-a_{v}}{x_{r}}$ is a root of the monic polynomial

$$
q(Y)=x_{-p r}\left(\left(a_{v}+x_{r} Y\right)^{p}-g^{p}\right)
$$

of degree $p$ with coefficients in $R\left[\frac{g^{p}-1}{x_{s}}\right]$. Hence an $R$ basis for $H$ consists of

$$
\left\{\left(\frac{g^{p}-1}{x_{s}}\right)^{i}\left(\frac{g-a_{v}}{x_{r}}\right)^{j}\right\},
$$

for $i, j=0, \ldots, p-1$.

For $\nu(1-v)$ sufficiently large we can assume that $v=1$; thus $a_{v}=1$. In this case the $R$-Hopf order $A_{1}(s, r)$ can be written as the Larson order

$$
H(s, r)=R\left[\frac{g^{p}-1}{x_{s}}, \frac{g-1}{x_{r}}\right] .
$$

Necessarily, we must have $p r \leq s$ (cf. [U2, §1.2]). As before, the algebra generator $\frac{g^{p}-1}{x_{s}}$ is a root of the monic polynomial

$$
p(X)=x_{-p s}\left(\left(1+x_{s} X\right)^{p}-1\right)
$$

of degree $p$ with coefficents in $R$, and the generator $\frac{g-1}{x_{r}}$ is a root of the monic polynomial

$$
q(Y)=x_{-p r}\left(\left(1+x_{r} Y\right)^{p}-g^{p}\right)
$$

of degree $p$ with coefficients in $R\left[\frac{g^{p}-1}{x_{s}}\right]$. It follows that an $R$ basis for the Larson order $H(s, r)$ consists of

$$
\left\{\left(\frac{g^{p}-1}{x_{s}}\right)^{i}\left(\frac{g-1}{x_{r}}\right)^{j}\right\},
$$

for $i, j=0, \ldots, p-1$.

Let $H=A_{v}(s, r)$ be an arbitrary order in $K C_{p^{2}}$. Since we supposed that $\zeta \in R$, the linear dual $H^{*}=\operatorname{Hom}_{R}(H, R)$ inherits the structure of a Hopf algebra order in $K C_{p^{2}}$ of the form

$$
H^{*}=A_{v^{\prime}}\left(r^{\prime}, s^{\prime}\right) \cong R\left[\frac{g^{p}-1}{x_{r^{\prime}}}, \frac{g-a_{v^{\prime}}}{x_{s^{\prime}}}\right]
$$

where $r^{\prime}=\frac{1}{p-1}-r, s^{\prime}=\frac{1}{p-1}-s$, and $v^{\prime}=1+\zeta-v$ (cf. [G, Remark 3.12], [U3, Thm 3.1.0]). We note that for the dual pair $A_{v}(s, r)$ and $A_{v^{\prime}}\left(r^{\prime}, s^{\prime}\right)$ we have either $p r \leq s$ or $p s^{\prime} \leq r^{\prime}$ (cf. [U2, Thms. 2.4, 2.5]). 
In general, an arbitrary order in $K C_{p^{2}}$ is either a Larson order $H(s, r)$, or a non-Larson order of the form $A_{v}(s, r)$.

Again, let $H$ denote an arbitrary $R$-Hopf order in $K C_{p^{2}}$.

Definition 1.0. An $H$-Galois extension of $R$ is a finitely generated projective $R$-algebra $S$ together with an $R$-algebra map

$$
\alpha: S \rightarrow S \otimes H
$$

satisfying the conditions

$$
\begin{gathered}
(\alpha \otimes 1) \alpha=(1 \otimes \Delta) \alpha, \\
(1 \otimes \epsilon) \alpha=I d_{S},
\end{gathered}
$$

with the map

$$
\gamma: S \otimes S \rightarrow S \otimes H
$$

given by

$$
\gamma(s \otimes t)=\sum_{(t)} s t_{(1)} \otimes t_{(2)}
$$

an isomorphism. Here we employ the Sweedler-like notation $\alpha(t)=\sum_{(t)} t_{(1)} \otimes t_{(2)}$, where $t_{(1)} \in S, t_{(2)} \in H$.

Definition 1.1. A finitely generated projective $R$-algebra $S$ is an $H$-Galois algebra if there exists an $H$-module map

$$
\beta: H \otimes S \rightarrow S
$$

satisfying

$$
\begin{gathered}
\beta(h \otimes 1)=\epsilon(h), \\
\beta(h \otimes x y)=\sum_{(h)} \beta\left(h_{(1)} \otimes x\right) \cdot \beta\left(h_{(2)} \otimes y\right),
\end{gathered}
$$

with the map

$$
H \otimes S \rightarrow \operatorname{End}_{R}(S), \quad h \otimes x \mapsto(y \mapsto x \cdot \beta(h \otimes y)),
$$

an isomorphism.

We note that $S$ is an $H$-Galois extension if and only if $S$ is an $H^{*}$-Galois algebra (cf. $[\mathrm{C}, \S 1])$.

Our goal in this paper is to characterize all $H$-Galois extensions $S$, where $H$ is an arbitrary $R$-Hopf algebra order in $K C_{p^{2}}$. Note that S. Hurley, [H], C. Greither, [G], and L. Roberts $[\mathrm{R}]$ all provide classifications of $H$-Galois extensions when $H$ is an arbitrary "Tate-Oort" order in $K C_{p}$. Our methods here generalize those of [G] and [R]. We realize that every $R$-Hopf order $H$ in $K C_{p^{2}}$ will give rise to a finite group scheme $S p_{R} H=H_{R-a l g}(H, \quad)$ of order $p^{2}$. Moreover, the cohomology group $H^{1}\left(R, S p_{R} H\right)$ can be identified with the collection of $H$-Galois extensions, up to $H$-comodule isomorphism. In other parlance, the group $H^{1}\left(R, S p_{R} H\right)$ corresponds to isomorphism classes of principal homogeneous spaces for $S p_{R} H$ over the base scheme $S p_{R} R$, and the affine algebras of these principal homogeneous spaces give rise to our $H$-Galois extensions. (Cf. [G, Intro.] and [M1, Ch.III, § 4].) 
Our plan is to calculate $H^{1}\left(R, S p_{R} H\right)$ and then give the algebraic structure of the corresponding $H$-Galois extensions. Our first step is to involve $S p_{R} H$ in a short exact sequence of group schemes. Specializing to the case where $H$ is a Larson order $H(s, r)$, we first resolve $\operatorname{SpH}(s, r)$ and then compute $H^{1}(R, S p H(s, r))$. Next we consider the class of non-Larson orders in $K C_{p^{2}}$, of the form $A_{v}(s, r)$. We then give a resolution of $S p A_{v}(s, r)$, and compute $H^{1}\left(R, S p A_{v}(s, r)\right)$.

\section{A Resolution of $S p_{R}(H(s, r))$}

To resolve $S p_{R}(H(s, r)$, we first need to define certain abelian group functors $\mathbf{E}_{s, r}$ and $\mathbf{E}_{p s, p r}$. We first define $\mathbf{E}_{s, r}$. Let $A$ be a commutative $R$-algebra, and let $G_{s, r}(A)$ be the subset of $U(A) \times U(A)$ defined

$$
G_{s, r}(A)=\left\{\left(u_{0}, u_{1}\right) \in U(A) \times U(A) \mid u_{0} \equiv 1\left(\bmod x_{s}\right) \text { and } u_{1} \equiv 1\left(\bmod x_{r}\right)\right\} .
$$

Here $a \equiv b\left(\bmod x_{s}\right)$ if and only if $a-b \in x_{s} A$. One easily checks that $G_{s, r}(A)$ forms a subgroup of $U(A) \times U(A)$ under coordinatewise multiplication. Moreover, for each element $\left(u_{0}, u_{1}\right) \in G_{s, r}(A)$ there is a pair $\left(w_{u_{0}}, w_{u_{1}}\right) \in A \times A$ with

$$
u_{0}=1+x_{s} w_{u_{0}} \quad \text { and } \quad u_{1}=1+x_{r} w_{u_{1}} .
$$

With this in mind, we define a subset $\mathbf{E}_{s, r}(A)$ of $A \times A$ as follows:

$$
\mathbf{E}_{s, r}(A)=\left\{\left(w_{u_{0}}, w_{u_{1}}\right) \in A \times A \mid w_{u_{0}}=\frac{u_{0}-1}{x_{s}} \quad \text { and } \quad w_{u_{1}}=\frac{u_{1}-1}{x_{r}}\right\},
$$

for some $\left(u_{0}, u_{1}\right) \in G_{s, r}(A)$.

By construction, we have a bijection of sets

$$
\rho: \mathbf{E}_{s, r}(A) \rightarrow G_{s, r}(A), \quad \rho\left(w_{u_{0}}, w_{u_{1}}\right)=\left(u_{0}, u_{1}\right) .
$$

In fact, we can put a group structure on $\mathbf{E}_{s, r}(A)$ so that $\rho$ is an isomorphism of abelian groups. For two elements $\left(w_{u_{0}}, w_{u_{1}}\right),\left(w_{v_{0}}, w_{v_{1}}\right) \in A \times A$ we simply define an operation

$$
\left(w_{u_{0}}, w_{u_{1}}\right) *\left(w_{v_{0}}, w_{v_{1}}\right)=\left(w_{u_{0} v_{0}}, w_{u_{1} v_{1}}\right),
$$

induced from the group structure of $G_{s, r}(A)$. We realize that $\mathbf{E}_{s, r}$ is a group functor from the category of commutative $R$-algebras to the category of abelian groups and is representable by the $R$-Hopf algebra

$$
B=R\left[T_{0}, T_{1},\left(1+x_{s} T_{0}\right)^{-1},\left(1+x_{r} T_{1}\right)^{-1}\right],
$$

where $T_{0}, T_{1}$ are indeterminates. Comultiplication in $B$ is the unique $R$-algebra map $\Delta: B \rightarrow B \otimes B$ which makes the elements $1+x_{s} T_{0}, 1+x_{r} T_{1}$ grouplike.

In a similar manner we define the abelian group functor $\mathbf{E}_{p s, p r}$. Let $A$ be any commutative $R$-algebra, and let

$$
\mathbf{E}_{p s, p r}(A)=\left\{\left(w_{u_{0}}, w_{u_{1}}\right) \in A \times A \mid w_{u_{0}}=\frac{u_{0}-1}{x_{p s}}, w_{u_{1}}=\frac{u_{1}-1}{x_{p r}}\right\},
$$

for some $\left(u_{0}, u_{1}\right) \in G_{p s, p r}(A)$ with

$$
G_{p s, p r}(A)=\left\{\left(u_{0}, u_{1}\right) \in U(A) \times U(A) \mid u_{0} \equiv 1\left(\bmod x_{p s}\right), u_{1} \equiv 1\left(\bmod x_{p r}\right)\right\} .
$$

We see that $\mathbf{E}_{p s, p r}$ is a group functor from the category of commutative $R$-algebras to the category of abelian groups, and is represented by the $R$-Hopf algebra

$$
R\left[T_{0}, T_{1},\left(1+x_{p s} T_{0}\right)^{-1},\left(1+x_{p r} T_{1}\right)^{-1}\right],
$$

with indeterminates $T_{0}, T_{1}$. 
Theorem 2.0. There is an epimorphism of flat sheaves $\Theta: \mathbf{E}_{s, r} \rightarrow \mathbf{E}_{p s, p r}$ whose kernel is the group scheme represented by the R-Hopf algebra $H(s, r)$.

Proof. Let $\Theta$ be the morphism on $\mathbf{E}_{s, r}$ defined

$$
\Theta(A)\left(\left(w_{u_{0}}, w_{u_{1}}\right)\right)=\left(p\left(w_{u_{0}}\right),\left(1+x_{s} w_{u_{0}}\right)^{-1} q\left(w_{u_{0}}, w_{u_{1}}\right)\right),
$$

with

$$
\begin{gathered}
p\left(T_{0}\right)=x_{-p s}\left(\left(1+x_{s} T_{0}\right)^{p}-1\right), \\
q\left(T_{0}, T_{1}\right)=x_{-p r}\left(\left(1+x_{r} T_{1}\right)^{p}-\left(1+x_{s} T_{0}\right)\right) .
\end{gathered}
$$

We first show that for an $R$-algebras $A$,

$$
\Theta(A)\left(\mathbf{E}_{s, r}(A)\right) \subseteq \mathbf{E}_{p s, p r}(A) .
$$

Let $\left(w_{u_{0}}, w_{u_{1}}\right) \in \mathbf{E}_{s, r}(A)$. Then

$$
\Theta(A)\left(w_{u_{0}}, w_{u_{1}}\right)=\Theta(A)\left(\frac{u_{0}-1}{x_{s}}, \frac{u_{1}-1}{x_{r}}\right)=\left(\frac{u_{0}^{p}-1}{x_{p s}}, \frac{u_{0}^{-1} u_{1}^{p}-1}{x_{p r}}\right) .
$$

Now since $s \leq \frac{1}{p-1}$ and $p r \leq s$,

$$
\frac{u_{0}-1}{x_{s}} \in A \Longrightarrow \frac{u_{0}^{p}-1}{x_{p s}} \in A,
$$

and

$$
\frac{u_{1}-1}{x_{r}} \in A \Longrightarrow \frac{u_{1}^{p}-1}{x_{p r}} \in A \Longrightarrow \frac{u_{0}^{-1} u_{1}^{p}-1}{x_{p r}} \in A .
$$

We next show that $\Theta$ is an epimorphism of $R$-group schemes in the flat topology. For an $R$-algebra $A$, let $\left(A \rightarrow A_{i}\right)_{i}$ be any flat covering of $A$. (We have identified affine open sets with their representing algebras, cf. [U1, Ch.2], [M1, pp. 46-66].) For $(a, b) \in \mathbf{E}_{p s, p r}(A)$, let $\left(a_{i}, b_{i}\right)$ be the image of $(a, b)$ under the induced maps

$$
\mathbf{E}_{p s, p r}(A) \rightarrow \mathbf{E}_{p s, p r}\left(A_{i}\right) .
$$

Now form the $A_{i}$-algebras

$A_{i}^{\prime}=A_{i}\left[T_{0}, T_{1},\left(1+x_{s} T_{0}\right)^{-1},\left(1+x_{r} T_{1}\right)^{-1}\right] /\left\langle p\left(T_{0}\right)-a_{i},\left(1+x_{s} T_{0}\right)^{-1} q\left(T_{0}, T_{1}\right)-b_{i}\right\rangle$.

By $\S 1, p\left(T_{0}\right)-a_{i}$ is monic of degree $p$ with coefficients in $A_{i}$ and $q\left(T_{0}, T_{1}\right)$ is monic, degree $p$ in $T_{1}$ with coefficents in $A_{i}\left[T_{0},\left(1+x_{s} T_{0}\right)^{-1}\right]$. It follows that the ideal generated by the coefficients of $\left(1+x_{s} T_{0}\right)^{-1} q\left(T_{0}, T_{1}\right)-b_{i} \in A_{i}\left[T_{0},\left(1+x_{s} T_{0}\right)^{-1}\right]\left[T_{1}\right]$ is all of $A_{i}\left[T_{0},\left(1+x_{s} T_{0}\right)^{-1}\right]$. Thus by [M1, p. 10, Remark 2.6], each map $A_{i} \rightarrow A_{i}^{\prime}$ is flat, and hence faithfully flat by [M1, Prop. 2.7]. Thus $\left(A \rightarrow A_{i}^{\prime}\right)_{i}$ is a flat covering of $A$.

Now let $x_{i}, y_{i}$ denote the images of $T_{0}, T_{1}$ respectively under the canonical map

$$
A_{i}\left[T_{0}, T_{1},\left(1+x_{s} T_{0}\right)^{-1},\left(1+x_{r} T_{1}\right)^{-1}\right] \rightarrow A_{i}^{\prime} .
$$

Then $\left(x_{i}, y_{i}\right) \in \mathbf{E}_{s, r}\left(A_{i}^{\prime}\right)$ with

$$
\Theta\left(A_{i}^{\prime}\right)\left(\left(x_{i}, y_{i}\right)\right)=\left(a_{i}, b_{i}\right)=\operatorname{res}_{A, A_{i}^{\prime}}((a, b))
$$

for all $i$, where $\operatorname{res}_{A, A_{i}^{\prime}}((a, b))$ is the image of $(a, b)$ under the induced maps

$$
\mathbf{E}_{p s, p r}(A) \rightarrow \mathbf{E}_{p s, p r}\left(A_{i}^{\prime}\right) ;
$$


hence $\Theta$ is an epimorphism of flat sheaves (group schemes) by [M1, p. 63, Thm. $2.15(\mathrm{c})]$.

We also have a canonical surjection of $R$-algebras:

$$
\begin{aligned}
& R\left[T_{0},\left(1+x_{s} T_{0}\right)^{-1}, T_{1},\left(1+x_{r} T_{1}\right)^{-1}\right] \longrightarrow \\
& \quad R\left[T_{0},\left(1+x_{s} T_{0}\right)^{-1}, T_{1},\left(1+x_{r} T_{1}\right)^{-1}\right] /\left\langle p\left(T_{0}\right),\left(1+x_{s} T_{0}\right)^{-1} q\left(T_{0}, T_{1}\right)\right\rangle \cong H(s, r),
\end{aligned}
$$

with $\bar{T}_{0}, \bar{T}_{1}$ identified with $\frac{g^{p}-1}{x_{s}}$ and $\frac{g-1}{x_{r}}$, respectively. Thus ker $\Theta=$ $S p_{R}(H(s, r))$.

It follows that the resulting short exact sequence of $R$-group schemes

$$
S p_{R}(H(s, r)) \rightarrow \mathbf{E}_{s, r} \stackrel{\Theta}{\longrightarrow} \mathbf{E}_{p s, p r}
$$

is a resolution of the group scheme $S p H$ where $H$ is an arbitrary Larson order in $K C_{p^{2}}$.

We are now in a position to prove our main theorem.

Theorem 2.1. Let $H=H(s, r)$ be an arbitrary Larson order in $K C_{p^{2}}$. Then the abelian group of $\mathrm{H}$-Galois extensions is isomorphic to the quotient group

$$
\mathbf{E}_{p s, p r}(R) / \mathbf{E}_{s, r}^{\Theta}(R),
$$

where the class $\left[\left(w_{u_{0}}, w_{u_{1}}\right)\right]$ corresponds to an $H$-Galois extension

$$
S=R\left[\frac{v_{0}-1}{x_{s}}, \frac{v_{1}-1}{x_{r}}\right]
$$

where $v_{0}^{p}=u_{0}, v_{1}^{p}=v_{0} u_{1}$. The comodule map $\rho: S \rightarrow S \otimes H(s, r)$ is given by

$$
\rho: v_{0} \mapsto v_{0} \otimes g^{p}, \quad \rho: v_{1} \mapsto v_{1} \otimes g .
$$

Proof. Using the given resolution of $\operatorname{SpH}(s, r)$, we employ the long exact sequence in cohomology yielding

$$
\begin{aligned}
H^{0}(R, S p H(s, r)) \longrightarrow H^{0}\left(R, \mathbf{E}_{s, r}\right) & \longrightarrow H^{0}\left(R, \mathbf{E}_{p s, p r}\right) \\
& \longrightarrow H^{1}(R, S p H(s, r)) \longrightarrow H^{1}\left(R, \mathbf{E}_{s, r}\right) \longrightarrow H^{1}\left(R, \mathbf{E}_{p s, p r}\right) \longrightarrow \cdots
\end{aligned}
$$

Note that

$$
H^{0}\left(R, \mathbf{E}_{s, r}\right)=\mathbf{E}_{s, r}(R)
$$

and

$$
H^{0}\left(R, \mathbf{E}_{p s, p r}\right)=\mathbf{E}_{p s, p r}(R) ;
$$

hence we have an exact sequence

$$
\mathbf{E}_{s, r}(R) \longrightarrow \mathbf{E}_{p s, p r}(R) \longrightarrow H^{1}(R, S p H(s, r)) \longrightarrow H^{1}\left(R, \mathbf{E}_{s, r}\right) .
$$

We claim that the last term $H^{1}\left(R, \mathbf{E}_{s, r}\right)$ is trivial. To this end suppose not, and let $S$ be a nontrivial $B$-Galois extension with structure map $\rho$. (Recall that $B$ is the representing algebra of $\mathbf{E}_{s, r}$.) By [G, Lemma II.1.6],

$$
\left\{x \in S \mid \rho(x) \in S \otimes_{R} R\left[T_{0},\left(1+x_{s} T_{0}\right)^{-1}\right]\right\}
$$

is a non-trivial $R\left[T_{0},\left(1+x_{s} T_{0}\right)^{-1}\right]$-Galois extension, contradicting [G, Proposition I.2.2], proving our claim. 
Now with $H^{1}\left(R, \mathbf{E}_{s, r}\right)=0$ we write the exact sequence

$$
\mathbf{E}_{s, r}(R) \stackrel{\Theta}{\longrightarrow} \mathbf{E}_{p s, p r}(R) \longrightarrow H^{1}(R, S p H(s, r)) .
$$

It follows that

$$
\mathbf{E}_{p s, p r}(R) / \mathbf{E}_{s, r}^{\Theta}(R) \cong H^{1}(R, S p H(s, r)) .
$$

Given an element $\left(w_{u_{0}}, w_{u_{1}}\right) \in \mathbf{E}_{p s, p r}(R)$ we construct the corresponding $H$-Galois extension $S$ by constructing the image of $\left(w_{u_{0}}, w_{u_{1}}\right)$ under the connecting homomorphism

$$
\delta: \mathbf{E}_{p s, p r}(R) \longrightarrow H^{1}(R, S p(H(s, r))) .
$$

Following the standard description of $\delta$ (cf. [CF, p.97], [Gi, Ch. III]), we recall our surjection in the flat topology

$$
\Theta: \mathbf{E}_{s, r} \longrightarrow \mathbf{E}_{p s, p r}
$$

There exists a finite extension $L / K$, with ring extension $A=O_{L} / R$ and an element $\left(w_{v_{0}}, w_{v_{1}}\right) \in \mathbf{E}_{s, r}(A)$ so that

$$
\Theta(A)\left(w_{v_{0}}, w_{v_{1}}\right)=\left(w_{u_{0}}, w_{u_{1}}\right) .
$$

In other words, the element $\left(v_{0}, v_{1}\right)$ satisfies

$$
v_{0}^{p}=u_{0}, \quad v_{0}^{-1} v_{1}^{p}=u_{1} .
$$

We now let $\delta\left(w_{u_{0}}, w_{u_{1}}\right)$ correspond to the pair $\left(v_{0}, v_{1}\right)$, forming the $R$-algebra

$$
S=R\left[\frac{v_{0}-1}{x_{s}}, \frac{v_{1}-1}{x_{r}}\right] .
$$

We claim that $S$ is an $H$-Galois extension contained in $O_{L} \subset L$. It is immediate that $S$ is finitely generated because by construction the generators $w_{v_{0}}=\frac{v_{0}-1}{x_{s}}$ and $w_{v_{1}}=\frac{v_{1}-1}{x_{r}}$ are in $A$. One can check by hand that $\rho$ is a comodule map, so it remains to show that $\rho$ induces a bijection

$$
\gamma_{\rho}: S \otimes S \longrightarrow S \otimes H
$$

Viewing the situation over $K$, we have that

$$
S \otimes K=K\left(v_{0}, v_{1}\right)=K\left(\sqrt[p]{u_{0}}, \sqrt[p]{u_{1} \sqrt[p]{u_{0}}}\right)=K\left(\sqrt[p^{2}]{u_{0} u_{1}^{p}}\right)=K\left(v_{1}\right)
$$

It follows that $S \otimes K$ is a $K C_{p^{2}}$-Galois extension with comodule map $\rho \otimes K$, by L. Robert's isomorphism

$$
U(K) / U(K)^{p^{2}} \cong H^{1}\left(K, \mu_{p^{2}, K}\right)
$$

which computes all $K C_{p^{2}}$-Galois extensions, cf. [R, p. 693]. Now since

$$
\gamma_{\rho \otimes K}:(S \otimes K) \otimes(S \otimes K) \longrightarrow(S \otimes K) \otimes K C_{p^{2}}
$$

is an isomorphism, then

$$
\gamma_{\rho}: S \otimes S \longrightarrow S \otimes H
$$

is an injection. Following [G, II.1.5], we can show that $\gamma_{\rho}$ is an bijection by showing that $\operatorname{disc}(S / R)=\operatorname{disc}(H / R)$. To this end, by [G, Prop. II.2.1]

$$
R\left[\frac{v_{0}-1}{x_{s}}\right]
$$


is an $H(s)$-Galois extension, where $H(s)$ is the Tate/Oort order $R\left[\frac{g^{p}-1}{x_{s}}\right]$. Thus by [G, Lemma II.1.5]

$$
\operatorname{disc}\left(R\left[\frac{g^{p}-1}{x_{s}}\right] / R\right)=\operatorname{disc}\left(R\left[\frac{v_{0}-1}{x_{s}}\right] / R\right) ;
$$

hence

$$
\operatorname{disc}\left(R\left[\frac{g^{p}-1}{x_{s}}\right] \otimes R\left[\frac{h-1}{x_{r}}\right] / R\right)=\operatorname{disc}\left(R\left[\frac{v_{0}-1}{x_{s}}\right] \otimes R\left[\frac{h-1}{x_{r}}\right] / R\right),
$$

where $h$ generates a cyclic group of order $p$. Additionally,

$$
\operatorname{disc}\left(R\left[\frac{v_{1}-1}{x_{r}}\right] / R\right)=\operatorname{disc}\left(R\left[\frac{h-1}{x_{r}}\right] / R\right) ;
$$

hence

$$
\operatorname{disc}\left(R\left[\frac{v_{0}-1}{x_{s}}\right] \otimes R\left[\frac{h-1}{x_{r}}\right] / R\right)=\operatorname{disc}\left(R\left[\frac{v_{0}-1}{x_{s}}\right] \otimes R\left[\frac{v_{1}-1}{x_{r}}\right] / R\right) .
$$

It follows that $\operatorname{disc}(S / R)=\operatorname{disc}(H / R)$ and hence $S$ is an $H$-Galois extension.

Moreover, if $\left(w_{u_{0}}, w_{u_{1}}\right)$ is so that $\left(w_{u_{0}}, w_{u_{1}}\right) \in \mathbf{E}_{s, r}^{\Theta}(R)$, then

$$
u_{0}=v_{0}^{p}, u_{1}=v_{0}^{-1} v_{1}^{p}
$$

where $\left(v_{0}, v_{1}\right) \in G_{s, r}(R)$. Now since $v_{1} \in K, S \otimes K$ will correspond to the trivial $K C_{p^{2}}$-Galois extension (again use L. Roberts classification). Since the canonical map $H^{1}(R, S p H(s, r)) \rightarrow H^{1}\left(K, \mu_{p^{2}, K}\right)$ is injective (cf. [M2], III.1.1), we conclude that $\left(w_{u_{0}}, w_{u_{1}}\right)$ corresponds to the trivial $H$-Galois extension iff $\left(w_{u_{0}}, w_{u_{1}}\right) \in$ $\mathbf{E}_{s, r}^{\Theta}(R)$. This completes the proof of the theorem.

We now turn our attention to calculating $H^{1}\left(R, S p A_{v}(s, r)\right)$.

\section{A Resolution of $S p_{R}\left(A_{v}(s, r)\right)$ When $A_{v}(s, r)$ is Not Larson}

To resolve $S p_{R}\left(A_{v}(s, r)\right)$, we first define the abelian group functor $\mathbf{W}_{s, r}$. We construct this functor by generalizing the method used to construct functors presented in [SS1, Prop. 2.2, Remark 2.3] and [SS2, §3]. In these papers, the authors have resolved

$$
S p A_{\zeta}(1 /(p-1), 1 /(p-1))=\operatorname{SpH}(0,0)^{*}=S p R C_{p^{2}}^{*},
$$

where $R C_{p^{2}}^{*}$ is identified with the maximal integral order in $K C_{p^{2}}$.

Let $F_{0}$ be the constant polynomial $F_{0}=1$, and let $F_{1}\left(T_{0}\right)$ be the polynomial in the indeterminate $T_{0}$ defined

$$
\begin{aligned}
F_{1}\left(T_{0}\right)= & \frac{1+\left(1+x_{s} T_{0}\right)+\cdots+\left(1+x_{s} T_{0}\right)^{p-1}}{p} \\
& +v\left(\frac{1+\zeta^{-p}\left(1+x_{s} T_{0}\right)+\cdots+\zeta^{-(p-1) p}\left(1+x_{s} T_{0}\right)^{p-1}}{p}\right)+\cdots \\
& +v^{p-1}\left(\frac{1+\zeta^{-(p-1) p}\left(1+x_{s} T_{0}\right)+\cdots+\zeta^{-(p-1)^{2} p}\left(1+x_{s} T_{0}\right)^{p-1}}{p}\right)
\end{aligned}
$$


Moreover, let

$$
\begin{gathered}
\alpha_{0}^{F}\left(T_{0}\right)=x_{s} T_{0}+F_{0}=x_{s} T_{0}+1, \\
\alpha_{1}^{F}\left(T_{0}, T_{1}\right)=x_{r} T_{1}+F_{1}\left(T_{0}\right),
\end{gathered}
$$

for $T_{0}, T_{1}$ indeterminate. Additionally, let

$$
\begin{gathered}
\beta_{0}^{F}\left(U_{0}\right)=\frac{U_{0}-1}{x_{s}}, \\
\Lambda_{0}^{F}\left(X_{0}, Y_{0}\right)=x_{s} X_{0} Y_{0}+X_{0}+Y_{0},
\end{gathered}
$$

for indeterminates $U_{0}, X_{0}, Y_{0}$. Finally, put

$$
\omega_{0}^{F}(i)=\beta_{0}^{F}\left(\zeta^{p i}\right)
$$

for $i \in \mathbf{Z}_{p}$. Here $\zeta^{p i}$ is defined as

$$
\zeta^{p i}=\zeta^{p i_{0}}
$$

where $i=\sum_{k \geq 0} i_{k} p^{k}$.

Lemma 3.0. Let $x_{s_{0}}=x_{s}$ and $x_{s_{1}}=x_{r}$. The family $\left\{F_{r}\right\}, r=0,1$, defined above satisfies the following conditions for each $r$ :

$$
\begin{gathered}
F_{r}(0)=1, \\
F_{r}\left(X_{0}\right) F_{r}\left(Y_{0}\right) \equiv F_{r}\left(\Lambda_{0}^{F}\left(X_{0}, Y_{0}\right)\right)\left(\bmod x_{s_{r}}\right), \\
F_{r}\left(\omega_{0}^{F}(1)\right) \equiv \zeta^{p^{1-r}}\left(\bmod x_{s_{r}}\right) .
\end{gathered}
$$

Proof. This can be verified directly. For example, if $p=2$, (ii) follows by the calculation

$$
\begin{aligned}
F_{1}( & \left.X_{0}\right) F_{1}\left(Y_{0}\right)-F_{1}\left(x_{s} X_{0} Y_{0}+X_{0}+Y_{0}\right) \\
= & \left(\frac{1+\left(1+x_{s} X_{0}\right)}{2}+\frac{v\left(1-\left(1+x_{s} X_{0}\right)\right)}{2}\right) \\
& \times\left(\frac{1+\left(1+x_{s} Y_{0}\right)}{2}+\frac{v\left(1-\left(1+x_{s} Y_{0}\right)\right)}{2}\right) \\
& -\left(\frac{1+\left(1+x_{s}\left(x_{s} X_{0} Y_{0}+X_{0}+Y_{0}\right)\right)}{2}+\frac{v\left(1-\left(1+x_{s}\left(x_{s} X_{0} Y_{0}+X_{0}+Y_{0}\right)\right)\right)}{2}\right) \\
= & \frac{x_{s}^{2} X_{0} Y_{0}}{4}+\frac{v^{2} x_{s}^{2} X_{0} Y_{0}}{4}-\frac{x_{s}^{2} X_{0} Y_{0}}{2} \\
\equiv & 0\left(\bmod x_{r}\right)
\end{aligned}
$$

since $\nu\left(1-v^{2}\right) \geq 2 s^{\prime}+r$.

Thus by [SS1, Remark 2.3] we can construct an $R$-group scheme $\mathbf{W}_{s, r}$ with representing algebra

$$
C=R\left[T_{0}, T_{1},\left(\alpha_{0}^{F}\left(T_{0}\right)\right)^{-1},\left(\alpha_{1}^{F}\left(T_{0}, T_{1}\right)\right)^{-1}\right] .
$$

Observe that property (ii) above guarantees a well-defined group law on $\mathbf{W}_{s, r}$ : Let $\left(m_{0}, m_{1}\right),\left(n_{0}, n_{1}\right) \in \mathbf{W}_{s, r}(A)$ for an $R$-algebra $A$. Then we define

$$
\left(m_{0}, m_{1}\right) *\left(n_{0}, n_{1}\right)=\left(m_{0}+n_{0}+x_{s} m_{0} n_{0}, m_{1} F_{1}\left(n_{0}\right)+n_{1} F_{1}\left(m_{0}\right)+x_{r} m_{1} n_{1}+y\right)
$$


where $y$ is some element of $A$ determined by the congruence in (ii). Moreover condition (i) implies an identity for $\mathbf{W}_{s, r}$.

We realize that there is an inclusion of group schemes

$$
S p\left(A_{v}(s, r)\right) \longrightarrow \mathbf{W}_{s, r}
$$

induced by the surjection

$$
C \longrightarrow C /\left\langle x_{-p s}\left(1-\alpha_{0}^{F}\left(T_{0}\right)^{p}\right), x_{-p r}\left(\alpha_{0}^{F}\left(T_{0}\right)-\alpha_{1}^{F}\left(T_{0}, T_{1}\right)^{p}\right)\right\rangle \cong A_{v}(s, r)
$$

with $\bar{T}_{0}, \bar{T}_{1}$ identified with $\frac{g^{p}-1}{x_{s}}$ and $\frac{g-a_{v}}{x_{r}}$, respectively. Moreover, there exists a commutative diagram

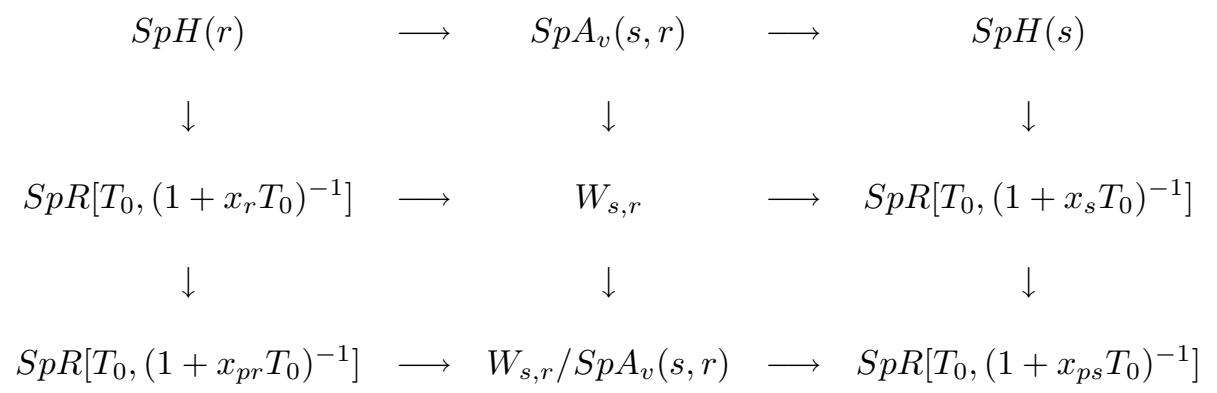

with all rows and columns s.e.s.'s. Here $H(s), H(r)$ denote Larson orders in $K C_{p}$. Thus the quotient $W_{s, r} / S p A_{v}(s, r)$ is a filtered group scheme of type ( $\left.p s, p r\right)$, with filtration given by $\operatorname{SpR}\left[T_{0},\left(1+x_{p s} T_{0}\right)^{-1}\right], S p R\left[T_{0},\left(1+x_{p r} T_{0}\right)^{-1}\right]$ (cf. [SS2, §3]). Thus by [SS2, Thm. 3.3]

$$
\mathbf{V}_{p s, p r}:=\mathbf{W}_{s, r} / S p\left(A_{v}(s, r)\right)=S p\left(R\left[T_{0}, T_{1},\left(\alpha_{0}^{G}\left(T_{0}\right)\right)^{-1},\left(\alpha_{1}^{G}\left(T_{0}, T_{1}\right)\right)^{-1}\right]\right),
$$

where

$$
\alpha_{0}^{G}\left(T_{0}\right)=1+x_{p s} T_{0}
$$

and

$$
\alpha_{1}^{G}\left(T_{0}, T_{1}\right)=G_{1}\left(T_{0}\right)+x_{p r} T_{1},
$$

for some polynomial $G_{1}\left(T_{0}\right) \in R\left[T_{0}\right]$ satisfying the conditions

$$
\begin{gathered}
G_{1}(0)=1, \\
G_{1}\left(X_{0}\right) G_{1}\left(Y_{0}\right) \equiv G_{1}\left(X_{0}+Y_{0}+x_{p s} X_{0} Y_{0}\right)\left(\bmod x_{p r}\right) .
\end{gathered}
$$

It follows that the resulting short exact sequence of $R$-group schemes

$$
S p_{R}\left(A_{v}(s, r)\right) \rightarrow \mathbf{W}_{s, r} \stackrel{\Psi}{\longrightarrow} \mathbf{V}_{p s, p r},
$$

where $\Psi$ is the canonical surjection, is a resolution of the group scheme $S p H$ where $H$ is an arbitrary non-Larson order in $K C_{p^{2}}$.

We are now in a position to prove our second main theorem.

Theorem 3.1. Let $H=A_{v}(s, r)$ be an arbitrary non-Larson order in $K C_{p^{2}}$. Then the abelian group of $\mathrm{H}$-Galois extensions is isomorphic to the quotient group

$$
\mathbf{V}_{p s, p r}(R) / \mathbf{W}_{s, r}^{\Psi}(R),
$$


where the class $\left[\left(t_{0}, t_{1}\right)\right]$ corresponds to an $H$-Galois extension $S$ of the form

$$
S=R\left[\frac{v_{0}-1}{x_{s}}, \frac{v_{1}-F_{1}\left(v_{0}^{\prime}\right)}{x_{r}}\right],
$$

where $v_{0}^{p}=\alpha_{0}^{G}\left(t_{0}\right), v_{0}^{-1} v_{1}^{p}=\alpha_{1}^{G}\left(t_{0}, t_{1}\right)$ and $v_{0}^{\prime}=\frac{v_{0}-1}{x_{s}}$. The comodule map $\rho$ : $S \rightarrow S \otimes A_{v}(s, r)$ is given by

$$
\begin{gathered}
\rho: v_{0} \mapsto v_{0} \otimes g^{p}, \\
\rho: v_{1} \mapsto v_{1} \otimes g .
\end{gathered}
$$

Proof. Using the given resolution of $S p\left(A_{v}(s, r)\right.$ ), we employ the long exact sequence in cohomology yielding

$$
\begin{aligned}
H^{0}\left(R, S p A_{v}(s, r)\right) & \longrightarrow H^{0}\left(R, \mathbf{W}_{s, r}\right) \longrightarrow H^{0}\left(R, \mathbf{V}_{p s, p r}\right) \\
\longrightarrow H^{1}\left(R, S p A_{v}(s, r)\right) \longrightarrow H^{1}\left(R, \mathbf{W}_{s, r}\right) \longrightarrow H^{1}\left(R, \mathbf{V}_{p s, p r}\right) & \longrightarrow \cdots
\end{aligned}
$$

Note that

$$
H^{0}\left(R, \mathbf{W}_{s, r}\right)=\mathbf{W}_{s, r}(R)
$$

and

$$
H^{0}\left(R, \mathbf{V}_{p s, p r}\right)=\mathbf{V}_{p s, p r}(R)
$$

hence we have an exact sequence

$$
\mathbf{W}_{s, r}(R) \longrightarrow \mathbf{V}_{p s, p r}(R) \longrightarrow H^{1}\left(R, S p A_{v}(s, r)\right) \longrightarrow H^{1}\left(R, \mathbf{W}_{s, r}\right)
$$

Now since $H^{1}\left(R, \mathbf{W}_{s, r}\right) \cong 0$, we obtain the isomorphism

$$
\mathbf{V}_{p s, p r}(R) / \mathbf{W}_{s, r}^{\Psi}(R) \cong H^{1}\left(R, S p A_{v}(s, r)\right) .
$$

Suppose $\left(t_{0}, t_{1}\right) \in \mathbf{V}_{p s, p r}(R)$. We will construct the corresponding $H$-Galois extension by computing the image of $\left(t_{0}, t_{1}\right)$ under the connecting homomorphism

$$
\delta: \mathbf{V}_{p s, p r}(R) \longrightarrow H^{1}\left(R, S p A_{v}(s, r)\right) \text {. }
$$

By [SS1, §2.3, p. 109], the canonical flat surjection $\Psi: \mathbf{W}_{s, r} \rightarrow \mathbf{V}_{p s, p r}$ can be defined via polynomials:

$$
\left(T_{0}, T_{1}\right) \mapsto\left(\Psi_{0}\left(T_{0}\right), \Psi_{1}\left(T_{0}, T_{1}\right)\right)
$$

where

$$
\begin{gathered}
\Psi_{0}\left(T_{0}\right)=\frac{\left(x_{s} T_{0}+1\right)^{p}-1}{x_{p s}}, \\
\Psi_{1}\left(T_{0}, T_{1}\right)=\frac{\left(x_{s} T_{0}+1\right)^{-1}\left(x_{r} T_{1}+F_{1}\left(T_{0}\right)\right)^{p}-G_{1}\left(\Psi_{0}\left(T_{0}\right)\right)}{x_{p r}} .
\end{gathered}
$$

Moreover, there exists a finite extension $L / K$ with $\operatorname{ring}$ extension $A=O_{L} / R$ and an element $\left(p_{0}, p_{1}\right) \in \mathbf{W}_{s, r}(A)$ so that

$$
\begin{gathered}
\Psi_{0}\left(p_{0}\right)=\frac{\left(x_{s} p_{0}+1\right)^{p}-1}{x_{p s}}=t_{0}, \\
\Psi_{1}\left(p_{0}, p_{1}\right)=\frac{\left(x_{s} p_{0}+1\right)^{-1}\left(x_{r} p_{1}+F_{1}\left(p_{0}\right)\right)^{p}-G_{1}\left(\Psi_{0}\left(p_{0}\right)\right)}{x_{p r}}=t_{1} .
\end{gathered}
$$


If we write $v_{0}=1+x_{s} p_{0}, v_{1}=F_{1}\left(p_{0}\right)+x_{r} p_{1}$, we have that $\delta\left(\left(t_{0}, t_{1}\right)\right)$ corresponds to the $H$-Galois extension

$$
S=R\left[\frac{v_{0}-1}{x_{s}}, \frac{v_{1}-F_{1}\left(v_{0}^{\prime}\right)}{x_{r}}\right],
$$

where $v_{0}^{\prime}=p_{0}$. This follows exactly as in Theorem 2.1, and we outline the proof. We first see that $S$ is finitely generated since $\left(p_{0}, p_{1}\right) \in \mathbf{W}_{s, r}(A)$. We then check that $S \otimes K$ is a $K C_{p^{2}}$-Galois extension, using L. Roberts isomorphism. Finally we conclude that $S$ is an $H$-Galois extension by showing that $\operatorname{disc}(S / R)=\operatorname{disc}(H / R)$.

Now suppose $\left(t_{0}, t_{1}\right) \in \mathbf{W}_{s, r}^{\Psi}(\mathrm{R})$. Then there exists $\left(p_{0}, p_{1}\right) \in \mathbf{W}_{s, r}(R)$ so that

$$
\begin{gathered}
t_{0}=\frac{\alpha_{0}^{F}\left(p_{0}\right)^{p}-1}{x_{p s}}, \\
t_{1}=\frac{\alpha_{0}^{F}\left(p_{0}\right)^{-1}\left(\alpha_{1}^{F}\left(p_{0}, p_{1}\right)\right)^{p}-G_{1}\left(t_{0}\right)}{x_{p r}} .
\end{gathered}
$$

Over $K$,

$$
S \otimes K=K\left(\alpha_{0}^{F}\left(p_{0}\right), \alpha_{1}^{F}\left(p_{0}, p_{1}\right)\right)=K\left(\sqrt[p^{2}]{\alpha_{1}^{G}\left(t_{0}, t_{1}\right)^{p} \alpha_{0}^{G}\left(t_{0}\right)}\right)=K\left(\alpha_{1}^{F}\left(p_{0}, p_{1}\right)\right),
$$

with $\alpha_{1}^{F}\left(p_{0}, p_{1}\right) \in K$. Hence $S \otimes K$ is the trivial $K C_{p^{2}}$-Galois extension. It follows that $S$ is the trivial $A_{v}(s, r)$-Galois extension iff the corresponding element $\left(t_{0}, t_{1}\right) \in$ $\mathbf{W}_{s, r}^{\Psi}(\mathrm{R})$. This completes the proof of the theorem.

\section{REFERENCES}

[CF] J.W.S. Cassels, A. Frohlich (eds.), Algebraic number theory, Academic Press, New York, (1967). MR 35:6500

[C] L.N. Childs, Taming wild extensions with Hopf algebras, Trans. Amer. Math. Soc. 304, No. 1(1987), 111-140. MR 89a:11119

[Gi] J. Giraud, Cohomologie non-abelienne, Columbia University, (1966).

[G] C. Greither, Extensions of finite group schemes, and Hopf Galois theory over a complete discrete valuation ring, Math. Z. 210, (1992), 37-67. MR 93f: 14024

$[\mathrm{H}] \quad$ S. Hurley, Galois objects with normal bases for free Hopf algebras of prime degree, J. Algebra 109, (1987), 292-318. MR 88k:13003

[M1] J. S. Milne, Etale Cohomology, Princeton University Press, Princeton, NJ (1980). MR $\mathbf{8 1 j}: 14002$

[M2] - Arithmetic duality theorems, Academic Press, Boston, (1986). MR 88e:14028

[R] L. Roberts, The flat cohomology of group schemes of order p, Amer. J. Math., 95, (1973), 688-702. MR 49:2741

[SS1] T. Sekiguchi, N. Suwa, Theories de Kummer-Artin-Schreier-Witt, Comptes Rendus de l'Acad. des Sci., 319, ser. I, 105-110, (1994). CMP 94:16

[SS2] _ On the unified Kummer-Artin-Schreier-Witt theory, Chuo University Preprint Series, no. 41, Chuo University, Bunkyoku, Tokyo, Japan (1994).

[U1] R.G.Underwood, Hopf algebra orders over a complete discrete valuation ring, their duals and extensions of $R$-groups, doctoral dissertation, State University of New York at Albany, (1992).

[U2],$R$-Hopf algebra orders in $K C_{p^{2}}, J$. Algebra, 169, (1994) 418-440. MR 95k:16055

[U3] - The valuative condition and $R$-Hopf algebra orders in $K C_{p^{3}}$, Amer. J. Math., 118, no. 4, (1996) 701-743. CMP 96:15

Department of Mathematics, Auburn University at Montgomery, Montgomery, AlABAMA 36117

E-mail address: underw@tango.aum.edu 